Article

\title{
Non-Extensivity of the Configurational Density Distribution in the Classical Microcanonical Ensemble
}

\author{
Jan Naudts * and Maarten Baeten \\ Departement Natuurkunde, Universiteit Antwerpen, Universiteitsplein 1, 2610 Antwerpen, Belgium \\ ^ Author to whom correspondence should be addressed; E-mail: jan.naudts@ua.ac.be
}

Received: 7 May 2009 / Accepted: 9 June 2009 / Published: 11 June 2009

\begin{abstract}
We show that the configurational probability distribution of a classical gas always belongs to the $q$-exponential family. One of the consequences of this observation is that the thermodynamics of the configurational subsystem is uniquely determined up to a scaling function. As an example we consider a system of non-interacting harmonic oscillators. In this example, the scaling function can be determined from the requirement that in the limit of large systems the microcanonical temperature of the configurational subsystem should coincide with that of the canonical ensemble. The result suggests that Renyi's entropy function is the relevant one rather than that of Tsallis.
\end{abstract}

Keywords: microcanonical ensemble; Tsallis entropy; Rényi entropy; $q$-exponential distribution

\section{Introduction}

Many-particle systems are usually studied in the canonical or grandcanonical ensemble. But for small systems the equivalence of ensembles breaks down and it becomes interesting to study the microcanonical ensemble. One of the intriguing questions in this context is whether phase transitions can occur in the way proposed by Dieter Gross in [1]. We will not consider this question here but use it as a motivation to study the thermodynamics of closed systems. The arguments of Gross are a mixture of statistical physics applied on microscopic models and of macroscopic thermodynamics. A more clean approach requires that the thermodynamic formalism is derived from the statistical theory rather than being used as a generally valid but unexplained addition. 
The present paper exploits the characteristic of classical systems that the coordinates and the conjugated momenta can be considered as two mutually interacting subsystems. By considering a subsystem one avoids the difficulty that the probability distribution of the microcanonical ensemble is a singular measure (a Dirac delta function). By integrating out the momenta, which is always possible for a Hamiltonian which is quadratic in the momenta, the configurational probability distribution results. To our surprise, this distribution function belongs to the $q$-exponential family, the definition of which is given in Section 3. A related observation, made 15 years ago [2], is that the probability distribution of a finite system in interaction with a heat bath is non-extensive in the sense of Tsallis [3]. The implications of our observation are important. It places non-extensive thermostatistics at the heart of statistical physics. In addition, many properties of the configurational subsystem can now be studied in a systematic way.

The present paper makes use of the knowledge that the probability distributions of non-extensive thermostatistics automatically induce the standard thermodynamical formalism. This means in particular that the thermodynamic entropy and the temperature of the configurational subsystem are fixed in a unique manner up to a monotonic function, which is referred to as the scaling function.

In the next Section we discuss various choices of the microcanonical entropy function. Section 3 introduces the notion of a $q$-exponential family of probability distributions. In Section 4 the configurational probability distribution is calculated. It is shown to belong to the $q$-exponential family. Section 5 deals with thermodynamic relations. Section 6 treats a simple example and discusses the scaling function. Finally, a short discussion follows in Section 7.

\section{Microcanonical entropies}

The entropy $S(U)$ which is most often used in the classical microcanonical ensemble is

$$
S(U)=k_{B} \ln \omega(U),
$$

where $\omega(U)$ is the $N$-particle density of states. The latter is given by

$$
\omega(U)=\frac{1}{h^{3 N}} \int_{\mathbb{R}^{3 N}} \mathrm{~d} \mathbf{p}_{1} \cdots \mathrm{d} \mathbf{p}_{N} \int_{\mathbb{R}^{3 N}} \mathrm{~d} \mathbf{q}_{1} \cdots \mathrm{d} \mathbf{q}_{N} \delta(U-H(\mathbf{q}, \mathbf{p})) .
$$

Here, $\mathbf{q}_{j}$ is the position of the $j$-th particle and $\mathbf{p}_{j}$ is the conjugated momentum, $H(\mathbf{q}, \mathbf{p})$ is the Hamiltonian. The constant $h$ is introduced for dimensional reasons. This definition goes back to Boltzmann's idea of equal probability of the microcanonical states and the corresponding well-known formula $S=k_{B} \ln W$, where $W$ is the number of microcanonical states. However, this choice of definition of entropy has some drawbacks. For instance, for the pendulum the entropy $S(U)$ as a function of internal energy $U$ is a piecewise convex function instead of a concave function [4]. The lack of concavity can be interpreted as a microcanonical instability $[1,5]$. But there is no physical reason why the pendulum should be classified as being instable at all energies.

The shortcomings of Boltzmann's entropy have been noticed long ago. A slightly different definition of entropy is $[6,7]$ (see also in [8] the reference to the work of A. Schlüter )

$$
S(U)=k_{B} \ln \Omega(U),
$$

where $\Omega(U)$ is the integral of $\omega(U)$ and is given by

$$
\Omega(U)=\frac{1}{h^{3 N}} \int_{\mathbb{R}^{3 N}} \mathrm{~d} \mathbf{p}_{1} \cdots \mathrm{d} \mathbf{p}_{N} \int_{\mathbb{R}^{3 N}} \mathrm{~d} \mathbf{q}_{1} \cdots \mathrm{d} \mathbf{q}_{N} \Theta(U-H(\mathbf{q}, \mathbf{p})) .
$$


Here, $\Theta(x)$ is Heaviside's function. An immediate advantage of (3) is that the resulting expression for the temperature $T$, defined by the thermodynamical formula

$$
\frac{1}{T}=\frac{\mathrm{d} S}{\mathrm{~d} U},
$$

coincides with the notion of temperature as used by experimentalists. Indeed, one finds

$$
k_{B} T=\frac{\Omega(U)}{\omega(U)} .
$$

For a harmonic oscillator the density of states $\omega(U)$ is a constant. Hence, (6) implies $k_{B} T=U$, as wanted. It is well-known that for classical monoatomic gases the r.h.s. of (6) coincides with twice the average kinetic energy per degree of freedom. This result is also derived below - see (28). Its significance is that the equipartition theorem, assigning $k_{B} T / 2$ to each degree of freedom, does hold for the kinetic energy also in the microcanonical ensemble. Quite often the average kinetic energy per degree of freedom is experimentally accessible and provides a unique way to measure accurately the temperature of the system.

But also (3) and (6) are subject to criticism. In small systems finite size corrections appear [8, 9] for a number of reasons. As argued in [9], the problem is not the equipartition of the kinetic energy over the various degrees of freedom, but the relation between temperature and kinetic energy.

\section{Generalized exponential family}

Recently, the notion of a generalized exponential family has been introduced both in the physics [1013] and in the mathematics [14-17] literature. It is shown in the next Section that the configurational probability distributions of a classical real gas in the microcanonical ensemble always belong to the $q$ exponential family, which is a special case of the generalized exponential family. A first observation in this direction was made in [13].

Fix a number $q$. The probability distribution $f_{\theta}(x)$ with parameter $\theta$ is said to belong to the $q$ exponential family if it can be written as

$$
f_{\theta}(x)=c(x) \exp _{q}(-\alpha(\theta)-\theta H(x))
$$

where the $q$-deformed exponential $[18,19]$ is defined by

$$
\exp _{q}(u)=[1+(1-q) u]_{+}^{1 /(1-q)}
$$

The notation $[u]_{+}=\max \{0, u\}$ is used. In (7) it is important that $H(x)$ and $c(x)$ do not depend on the parameter $\theta$ and that the normalization constant $\alpha(\theta)$ does not depend on $x$. In the limit $q=1$ the $q$ exponential function reduces to the natural exponential function. The notion of the $q$-exponential family then reduces to the standard notion of an exponential family.

Distributions belonging to the $q$-exponential family share a number of properties which make it attractive to work with these distributions. See for instance [13]. These properties are well-known to physicists because the Boltzmann-Gibbs distribution

$$
f_{\beta}(x)=\frac{1}{Z} \exp (-\beta H(x))
$$


belongs to the standard exponential family, which corresponds with the choice $q=1$. In particular, when $H(x)$ is the energy of a mechanical system, and $f_{\theta}(x)$ belongs to the $q$-exponential family, then there is a unique way to fit the statistical model into the context of thermodynamics.

\section{The configurational probability distribution}

A classical model of $N$ particles is determined by the Hamiltonian

$$
H(\mathbf{q}, \mathbf{p}) \equiv H\left(\mathbf{q}_{1}, \mathbf{q}_{2}, \cdots \mathbf{q}_{N}, \mathbf{p}_{1}, \mathbf{p}_{2}, \cdots \mathbf{p}_{N}\right)
$$

where $\mathbf{q}_{j}$ is the position of the $j$-th particle and $\mathbf{p}_{j}$ is the conjugated momentum. The microcanonical ensemble is then described by the singular probability density function

$$
f_{U}(\mathbf{q}, \mathbf{p})=\frac{1}{\omega(U)} \delta(U-H(\mathbf{q}, \mathbf{p}))
$$

where $\delta(\cdot)$ is Dirac's delta function. The normalization is so that

$$
1=\frac{1}{h^{3 N}} \int_{\mathbb{R}^{3 N}} \mathrm{~d} \mathbf{p}_{1} \cdots \mathrm{d} \mathbf{p}_{N} \int_{\mathbb{R}^{3 N}} \mathrm{~d} \mathbf{q}_{1} \cdots \mathrm{d} \mathbf{q}_{N} f_{U}(\mathbf{q}, \mathbf{p}) .
$$

The particles are enclosed in a box with volume $V$. For simplicity, we take only one conserved quantity into account, namely the total energy. Its value is fixed to $U$.

In the simplest case the Hamiltonian is of the form

$$
H(\mathbf{q}, \mathbf{p})=\frac{1}{2 m} \sum_{j=1}^{N}\left|\mathbf{p}_{j}\right|^{2}+\mathcal{V}(\mathbf{q})
$$

where $\mathcal{V}(\mathbf{q})$ is the potential energy due to interaction among the particles and between the particles and the walls of the system. It is then possible to integrate out the momenta. This leads to the configurational probability distribution, which is given by

$$
f_{U}^{\text {conf }}(\mathbf{q})=\frac{1}{h^{3 N}} \int_{\mathbb{R}^{3 N}} \mathrm{~d} \mathbf{p}_{1} \cdots \mathrm{d} \mathbf{p}_{N} f_{U}(\mathbf{q}, \mathbf{p}) .
$$

The normalization is so that

$$
1=\int_{\mathbb{R}^{3 N}} \mathrm{~d} \mathbf{q}_{1} \cdots \mathrm{d} \mathbf{q}_{N} f_{U}^{\text {conf }}(\mathbf{q})
$$

Let $B(N)$ denote the volume of a sphere of radius 1 in dimension $N$. A short calculation gives

$$
\begin{aligned}
f_{U}^{\text {conf }}(\mathbf{q}) & =\frac{1}{h^{3 N}} \frac{1}{\omega(U)} \int_{\mathbb{R}^{3 N}} \mathrm{~d} \mathbf{p}_{1} \cdots \mathrm{d} \mathbf{p}_{N} \delta(U-H(\mathbf{q}, \mathbf{p})) \\
& =\frac{1}{h^{3 N}} \frac{1}{\omega(U)} \frac{\mathrm{d}}{\mathrm{d} U} \int_{\mathbb{R}^{3 N}} \mathrm{~d} \mathbf{p}_{1} \cdots \mathrm{d} \mathbf{p}_{N} \Theta\left(U-\mathcal{V}(\mathbf{q})-\frac{1}{2 m} \sum_{j=1}^{N}\left|\mathbf{p}_{j}\right|^{2}\right) \\
& =\frac{1}{h^{3 N}} \frac{1}{\omega(U)}(2 m)^{3 N / 2} B(3 N) \frac{\mathrm{d}}{\mathrm{d} U}[U-\mathcal{V}(\mathbf{q})]_{+}^{3 N / 2} \\
& =\frac{1}{2 h^{3 N}} \frac{3 N}{\omega(U)}(2 m)^{3 N / 2} B(3 N)[U-\mathcal{V}(\mathbf{q})]_{+}^{\frac{3}{2} N-1}
\end{aligned}
$$




$$
=c_{N} \exp _{q}(-\alpha(\theta)-\theta \mathcal{V}(\mathbf{q}))
$$

with

$$
\begin{aligned}
c_{N} & =\left(\frac{2 m}{h^{2}}\right)^{3 N / 2}, \\
\theta & =\frac{1}{1-q} \frac{1}{[\Gamma(3 N / 2) \omega(U)]^{1-q}}, \\
\alpha(\theta) & =\frac{3}{2} N-1-\theta U, \\
q & =1-\frac{2}{3 N-2} .
\end{aligned}
$$

For convenience we assume here that $\omega(U)$ is a strictly increasing function of $U$ so that it can be inverted to obtain $U$ as a function of $\theta$. One concludes from (16) that the configurational density function $f_{U}^{\text {conf }}(\mathbf{q})$ of a classical gas in the microcanonical ensemble with parameter $U$ always belongs to the $q$-exponential family with the constant $q$ given by (17).

\section{Dual identities}

It is well-known that the $q$-exponential distribution optimizes the Tsallis entropy [20] and that together with the configurational energy $U^{\text {conf }}$ it satisfies the thermodynamic duality relations [10]. As shown below, these identities imply the statement that the ratio $\Omega(U) / \omega(U)$ equals the average kinetic energy.

Of course, also any monotonically increasing function of the Tsallis entropy will be optimized by the same probability distributions. In particular, Rényi’s alpha-entropy [21,22], given by

$$
I_{\alpha}(f)=\frac{1}{1-\alpha} \ln c_{N} \int_{\mathbb{R}^{3 N}} \mathrm{~d} \mathbf{q}_{1} \cdots \mathrm{d} \mathbf{q}_{N}\left(\frac{f(\mathbf{q})}{c_{N}}\right)^{\alpha},
$$

is such an equivalent entropy function (note that this expression slightly differs from the standard one; its justification is found in [23]). For that reason we will write the configurational entropy $S^{\text {conf }}$ as an unknown monotonic function of a quantity $\tilde{S}^{\text {conf }}$, where the latter is obtained by maximizing the entropy function $I(f)$.

An appropriate way of writing Tsallis' entropy $I(f)$ is [13] (assume $k_{B}=1$ for convenience)

$$
I(f)=-c_{N} \int_{\mathbb{R}^{3 N}} \mathrm{~d} \mathbf{q}_{1} \cdots \mathrm{d} \mathbf{q}_{N} F\left(\frac{1}{c_{N}} f(\mathbf{q})\right)
$$

with

$$
F(u)=\int_{0}^{u} \mathrm{~d} v \ln _{q}(v)=\frac{u}{1-q}\left(\frac{1}{2-q} u^{1-q}-1\right) .
$$

Using $(16,17)$ one obtains

$$
\begin{aligned}
\tilde{S}^{\text {conf }} & \equiv I\left(f_{U}^{\text {conf }}\right) \\
& =-\frac{1}{1-q} \int_{\mathbb{R}^{3 N}} \mathrm{~d} \mathbf{q}_{1} \cdots \mathrm{d} \mathbf{q}_{N} f_{U}^{\text {conf }}(\mathbf{q})\left(\frac{1}{2-q}\left(\frac{1}{c_{N}} f_{U}^{\text {conf }}(\mathbf{q})\right)^{1-q}-1\right) \\
& =\frac{1}{2-q}\left(1+\alpha(\theta)+\theta U^{\text {conf }}\right)
\end{aligned}
$$




$$
=\frac{1}{1-q}-\frac{\theta}{2-q} U^{\mathrm{kin}},
$$

with $U^{\text {kin }}=U-U^{\text {conf }}$.

The corresponding Massieu function is then given by

$$
\begin{aligned}
\tilde{\Phi}(\theta) & =\tilde{S}^{\mathrm{conf}}-\theta U^{\mathrm{conf}} \\
& =\frac{1}{1-q}-\frac{\theta U}{2-q}-\frac{1-q}{2-q} \theta U^{\mathrm{conf}}
\end{aligned}
$$

Using the dual identities $[10,13]$

$$
\frac{\mathrm{d} \tilde{\Phi}}{\mathrm{d} \theta}=-U^{\mathrm{conf}} \quad \text { and } \quad \frac{\mathrm{d} \tilde{S}^{\mathrm{conf}}}{\mathrm{d} U^{\operatorname{conf}}}=\theta
$$

one obtains

$$
U^{\text {conf }}=U+(1-q) \theta \frac{\mathrm{d} U^{\operatorname{conf}}}{\mathrm{d} \theta}+\theta \frac{\mathrm{d} U}{\mathrm{~d} \theta}
$$

and

$$
(1-q) \theta=-\left[U^{\operatorname{kin}} \frac{\mathrm{d} \theta}{\mathrm{d} U}+\theta\right] \frac{\mathrm{d} U}{\mathrm{~d} U^{\operatorname{conf}}}
$$

Since both identities imply the same result we continue with one of them. The latter can be written as

$$
\frac{\mathrm{d} U^{\text {conf }}}{\mathrm{d} U}=\frac{\omega^{\prime}(U)}{\omega(U)} U^{\mathrm{kin}}-\frac{1}{1-q} .
$$

Use this result to calculate

$$
\begin{aligned}
\frac{\mathrm{d}}{\mathrm{d} U} \omega(U) U^{\mathrm{kin}} & =\omega^{\prime}(U) U^{\mathrm{kin}}+\omega(U)\left[1-\frac{\mathrm{d} U^{\text {conf }}}{\mathrm{d} U}\right] \\
& =\frac{2-q}{1-q} \omega(U)=\frac{3 N}{2} \omega(U) .
\end{aligned}
$$

By integrating this expression one obtains the average kinetic energy

$$
U^{\mathrm{kin}}=\frac{3 N}{2} \frac{\Omega(U)}{\omega(U)} .
$$

This expression gives the relation between the average kinetic energy and the total energy $U$ via the density of states $\omega(U)$ and its integral $\Omega(U)$. The integration constant must be taken so that $\Omega(U)=0$ when $U=U_{\min }$ (implying that the kinetic energy vanishes in the ground state).

\section{Example}

Consider a set of $3 N$ harmonic oscillators. The potential energy equals

$$
\mathcal{V}(\mathbf{q})=\frac{1}{2} m \sum_{j=1}^{3 N} \omega_{j}^{2} \mathbf{q}_{j}^{2} .
$$


One calculates

$$
\begin{aligned}
\Omega(U)= & \frac{1}{h^{3 N}} \int_{\mathbb{R}^{3 N}} \mathrm{~d} \mathbf{p}_{1} \cdots \mathrm{d} \mathbf{p}_{3 N} \int_{\mathbb{R}^{3 N}} \mathrm{~d} \mathbf{q}_{1} \cdots \mathrm{d} \mathbf{q}_{3 N} \\
& \times \Theta\left(U-\frac{1}{2 m} \sum_{j} \mathbf{p}_{j}^{2}-\frac{1}{2} m \sum_{j} \omega_{j}^{2} \mathbf{q}_{j}^{2}\right) \\
= & \frac{1}{(3 N) ! \prod_{j=1}^{3 N} \omega_{j}}\left(\frac{4 \pi U}{h}\right)^{3 N} .
\end{aligned}
$$

From (17) and (30) now follows

$$
\frac{1}{\theta}=(1-q)\left[\frac{\Gamma(3 N / 2)}{\Gamma(3 N)} \frac{1}{\prod_{j=1}^{3 N} \omega_{j}}\left(\frac{4 \pi}{h}\right)^{3 N} U^{3 N-1}\right]^{1-q} .
$$

Using Stirling's approximation one obtains

$$
\frac{\Gamma(3 N / 2)}{\Gamma(3 N)} \sim \sqrt{2}\left(\frac{\mathrm{e}}{6 N}\right)^{3 N / 2}
$$

so that, assuming that all $\omega_{j}$ are equal to some $\omega$,

$$
\frac{1}{\theta} \sim \mathrm{e}\left(\frac{4 \pi}{h \omega} \frac{U}{3 N}\right)^{2}
$$

On the other hand, the inverse temperature $\beta$ of the canonical ensemble with $3 N$ degrees of freedom satisfies

$$
\frac{1}{\beta}=\frac{U}{3 N}
$$

The requirement that the canonical temperature coincides with the microcanonical temperature as obtained from (5) now determines the monotonic function which relates the thermodynamic $S^{\text {conf }}$ to the quantity $\tilde{S}^{\text {conf }}$. From this requirement follows that

$$
S^{\mathrm{conf}}=3 N \ln 2 U^{\mathrm{conf}}+A,
$$

for some constant $A$. On the other hand, (33) implies that

$$
\tilde{S}^{\text {conf }} \sim-\frac{1}{\mathrm{e}}\left(\frac{h \omega}{4 \pi}\right)^{2} \frac{(3 N)^{2}}{U^{\text {conf }}}+\text { constant. }
$$

Therefore the relation between $S^{\text {conf }}$ and $\tilde{S}^{\text {conf }}$ is logarithmic. More precisely, $S^{\text {conf }}=\xi\left(\tilde{S}^{\text {conf }}\right)$, with $\xi(x)$ of the form $\xi(x)=-3 N \ln (B-x)+C$, with constants $B$ and $C$. This suggests that Rényi's entropy function is the right one to start with. Indeed, let $\alpha=2-q$. The relation between Rényi's $I_{\alpha}(f)$ and $I(f)$, as given by (19), is $I_{\alpha}(f)=\xi(I(f))$ with

$$
\xi(x)=-\frac{1}{1-q} \ln [(2-q)(1-(1-q) x)] .
$$

Take the constant in (36) equal to $1 /(1-q)$. Then one obtains

$$
S^{\mathrm{conf}} \equiv \xi\left(\tilde{S}^{\mathrm{conf}}\right) \simeq\left(\frac{3 N}{2}-1\right)\left[\ln \frac{U^{\mathrm{conf}}}{6 N}+\text { constant }\right] .
$$


This yields

$$
\frac{\mathrm{d} S^{\operatorname{conf}}}{\mathrm{d} U^{\operatorname{conf}}}=\left(\frac{3 N}{2}-1\right) \frac{1}{U^{\operatorname{conf}}}=\frac{3 N-2}{U},
$$

which is an acceptable relation for the inverse temperature $\beta$, because it predicts that the total energy $U$ is an extensive quantity.

\section{Discussion}

The main purpose of the present paper is to point out that the configurational probability distribution of a classical gas always belongs to the $q$-exponential family. The non-extensivity parameter $q$ is given by

$$
\frac{1}{1-q}=\frac{3}{2} N-1
$$

where $N$ is the number of particles. The latter expression has appeared quite often in the literature, see for instance [2, 24, 25]. Reference [2] considers a finite system in contact with a finite heat bath. Making some assumptions this leads to a Tsallis canonical distribution, which can be written as a $q$-exponential family. Next the assumption is made that the heat bath consists of harmonic oscillators. This assumption is fulfilled in the present case because the heat bath consists of the kinetic degrees of freedom. Ref. [24] deals with the ideal gas in the Tsallis canonical ensemble. Ref. [25] studies homogeneous Hamiltonian systems in the same ensemble.

The main consequence of our observation is that the subsystem of configurational degrees of freedom can be described using standard thermodynamics involving the entropy $S^{\text {conf }}$ of the configurational subsystem. The thermodynamic relation

$$
k_{B} \beta=\frac{\mathrm{d} S^{\text {conf }}}{\mathrm{d} U^{\text {conf }}}
$$

then defines the microcanonical inverse temperature $\beta$. The same thermodynamic relation can be used to rederive a known result, which states that the average kinetic energy $U^{\text {kin }}$ of a classical gas in the microcanonical ensemble can be obtained from the density of states $\omega(U)$ and its integral $\Omega(U)$ - see (28).

Note that the probability distributions determine the entropy function up to a monotonically increasing function $\xi(x)$. This is so because the same entropy function is maximized for all values of the parameters. To cope with the non-uniqueness we used the notations $\tilde{S}$ instead of $S$ and $\theta$ instead of $\beta$ for the entropy, respectively the inverse temperature, because the function $\xi$ which relates $\tilde{S}$ to the thermodynamic $S$ is not known a priori. However, in the limit of a large system $(N \rightarrow \infty)$ the non-extensivity parameter goes to 1 and the configurational probability distribution approximates a Boltzmann-Gibbs distribution. It is then obvious that $\xi(x)$ should be chosen in such a way that the inverse temperature $\theta$ becomes the inverse temperature $\beta$ of the canonical ensemble.

For the example of $3 N$ non-interacting harmonic oscillators we calculate all quantities explicitly and obtain an explicit expression for the scaling function $\xi(x)$. It turns out that the relevant entropy function, which equals the thermodynamic entropy when evaluated in equilibrium, is not that of Tsallis 
but rather that of Rényi. From the point of view of Jaynes' maximum entropy principle both are of course equivalent. But in the context of thermodynamics only one of the two can yield correct values for the temperature. Note that the preference for the Rényi entropy is based here on one simple example. Further investigations are needed to establish this result in a more abstract setting.

In conclusion, we did not solve the problem of fixing the right expression for the microcanonical entropy $S$. But a good candidate for the configurational contribution $S^{\text {conf }}$ is the value of Rényi's entropy function evaluated at the equilibrium value of the configurational probability distribution.

\section{Acknowledgments}

We are grateful to Prof. Constantino Tsallis for pointing out reference [25].

\section{References}

1. Gross, D.H.E. Microcanonical Thermodynamics: Phase transitions in 'small' systems, Vol. 66, Lecture Notes in Physics. World Scientific, Singapore, 2001.

2. Plastino, A.R.; Plastino, A. From Gibbs microcanonical ensemble to Tsallis generalized canonical distribution. Phys. Lett. A 1994, 193, 140-143.

3. Tsallis, C. Nonextensive statistical mechanics: construction and physical interpretation. In Nonextensive Entropy; Gell-Mann, M.; Tsallis, C., Eds. Oxford University Press: Oxford, UK, 2004, pp. $1-53$.

4. Naudts, J. Boltzmann entropy and the microcanonical ensemble. Europhys. Lett. 2005, 69, 719724.

5. Gross, D. Statistical decay of very hot nuclei, the production of large clusters. Rep. Progr. Phys. 1990, 53, 605-658.

6. Schlüter, A. Zur Statistik klassischer Gesamtheiten. Z. Naturforschg. 1948, 3a, 350-360.

7. Pearson, E.M.; Halicioglu, T.; Tiller, W.A. Laplace-transform technique for deriving thermodynamic equations from the classical microcanonical ensemble. Phys. Rev. A 1985, 32, 3030-3039.

8. Shirts, R.B.; Burt, S.R.; Johnson, A.M. Periodic boundary condition induced breakdown of the equipartition principle and other kinetic effects of finite sample size in classical hard-sphere molecular dynamics simulation. J. Chem. Phys. 2006, 125, 164102.

9. Uline, M.J.; Siderius, D.W.; Cortib, D.S. On the generalized equipartition theorem in molecular dynamics ensembles and the microcanonical thermodynamics of small systems. J. Chem Phys. 2008, $128,124301$.

10. Naudts, J. Escort operators and generalized quantum information measures. Open Systems and Information Dynamics 2005, 12, 13-22.

11. Naudts, J. Parameter estimation in nonextensive thermostatistics. Physica A 2006, 365, 42-49.

12. Ohara, A.; Wada, T. Information Geometry of $q$-Gaussian Densities and Behaviors of Solutions to Related Diffusion Equations. arXiv:0810.0624.

13. Naudts, J. The $q$-exponential family in statistical physics. Cent. Eur. J. Phys. 2008, http://dx.doi.org/10.2478/s11534-008-0150-x. 
14. Naudts, J. Estimators, escort probabilities, and phi-exponential families in statistical physics. $J$. Ineq. Pure Appl. Math. 2004, 5, 102.

15. Grünwald, P.D.; Dawid, A.P. Game theory, maximum entropy, minimum discrepancy and robust bayesian decision theory. Ann. Stat. 2004, 32, 1367.

16. Eguchi, S. Information geometry and statistical pattern recognition. Sugaku Expositions 2006, 19, 197-216.

17. Naudts, J. Generalised Exponential Families and Associated Entropy Functions. Entropy 2008, $10,131-149$.

18. Tsallis, C. What are the numbers that experiments provide? Quimica Nova 1994, 17, 468.

19. Naudts, J. Deformed exponentials and logarithms in generalized thermostatistics. Physica A 2002, 316, 323-334.

20. Tsallis, C. Possible Generalization of Boltzmann-Gibbs Statistics. J. Stat. Phys. 1988, 52, 479487.

21. Rényi, A. On the foundations of information theory. Rev. Inst. Internat. Stat. 1965, 33, 1 (reprinted in [22], pp. 304-318).

22. Selected Papers of Alfréd Rényi, Vol. 2. Akadémiai Kiadó, 1976.

23. Czachor, M.; Naudts, J. Thermostatistics based on Kolmogorov-Nagumo averages: Unifying framework for extensive and nonextensive generalizations. Phys. Lett. A 2002, 298, 369-374.

24. Abe, S.; Martinez, S.; Pennini, F.; Plastino, A. Classical gas in nonextensive optimal Lagrange multipliers formalism. Phys. Lett. A 2001, 278, 249-254.

25. Adib, A.B.; Moreira, A.A.; Andrade Jr., J.S.; Almeida, M.P. Tsallis thermostatistics for finite systems: a Hamiltonian approach. Physica A 2003, 322, 276-284.

(c) 2009 by the authors; licensee Molecular Diversity Preservation International, Basel, Switzerland. This article is an open-access article distributed under the terms and conditions of the Creative Commons Attribution license http://creativecommons.org/licenses/by/3.0/. 